\title{
EFEITOS DE REGULADORES VEGETAIS NA QUALIDADE DE UVAS 'NIAGARA ROSADA' NA REGIÃO NOROESTE DO ESTADO DE SÃO PAULO ${ }^{1}$
}

\author{
RENATO VASCONCELOS BOTELHO² ${ }^{2}$ ERASMO JOSÉ PAIOLI PIRES ${ }^{3}$, MAURILO MONTEIRO TERRA $^{3}$
}

\begin{abstract}
RESUMO - Na região Noroeste do Estado de São Paulo, a uva 'Niagara Rosada' é produzida entre junho e novembro, como alternativa ao período de colheita das regiões vitícolas tradicionais. No entanto, devido às condições edafoclimáticas, as bagas e cachos são pequenos, o que é desfavorável à comercialização. Neste contexto, um experimento foi conduzido em vinhedo localizado em Junqueirópolis (SP), na região da Nova Alta Paulista, com o objetivo de se estudar os efeitos de reguladores vegetais nas características dos cachos e bagas de uvas 'Niagara Rosada'. Para os tratamentos, utilizou-se de thidiazuron a 5 ou $10 \mathrm{mg} . \mathrm{L}^{-1} \mathrm{e}$ ácido giberélico a $35 \mathrm{mg} . \mathrm{L}^{-1}$, associados ou não. Foram realizadas 1; 2 ou 3 aplicações semanais, a partir dos 14 dias após o pleno florescimento, mediante imersão dos cachos em solução contida em recipiente. As variáveis avaliadas foram: massa, comprimento e largura dos cachos e bagas; massa dos engaços, número de bagas; relação comprimento/largura das bagas; número de sementes; teor de sólidos solúveis totais, acidez total titulável, pH e relação SST/ATT do mosto. Dentre os diferentes tratamentos testados, 2 aplicações de thidiazuron a 5mg.L ${ }^{-1}$ foi o mais promissor para utilização comercial, levando a um incremento de 33,7\% da massa dos cachos e de 22,4\% da massa das bagas, sem alterar o teor de sólidos solúveis totais, a acidez total titulável, o pH e a relação SST/ATT do mosto. Aplicações de reguladores vegetais também aumentaram a massa dos engaços e a largura das bagas.
\end{abstract}

Termos para indexação: Vitis labrusca, videira, frutos, ácido giberélico, thidiazuron.

\section{EFFECTS OF PLANT REGULATORS ON THE QUALITY OF 'NIAGARA ROSADA' GRAPES IN THE NORTHWESTERN OF SÃO PAULO STATE}

\begin{abstract}
In the northwestern of São Paulo State, 'Niagara Rosada' grapes are harvest off-season in traditional vine-growing areas, between June and November. However, because of soil and climate conditions, clusters and berries are small, what is unfavorable for trading. In this context, a trial was carried out in a vineyard located in Junqueirópolis-SP, Alta Nova Paulista region, with the objective of studying the effects of plant regulators on cluster and berry characteristics of 'Niagara Rosada' grapes. For treatments, it was used thidiazuron at 5 or $10 \mathrm{mg} . \mathrm{L}^{-1}$ and gibberellic acid at $35 \mathrm{mg} . \mathrm{L}^{-1}$, associated or not. It was realized 1, 2 or 3 weekly applications, beginning 14 days after full bloom, by dipping cluster in solution contained in a recipient. The following variables were evaluated: weight, length and width of clusters and berries; weight of rachis; number of berries; number of seeds; total soluble solids content; total titrable acidity, $\mathrm{pH}$ and TSS/TTA ratio of juice. Between the different treatments tested, 2 applications of thidiazuron at $5 \mathrm{mg} . \mathrm{L}^{-1}$, was the most promissory for commercial use, leading to increase cluster weight in $33,7 \%$, and berry weight in $22,4 \%$; without changing total soluble solids content, total titrable acidity, $\mathrm{pH}$ and TSS/TTA ratio of juice. Plant regulators applications also improved rachis weight and berry width.
\end{abstract}

Index terms: Vitis labrusca, vine, fruits, gibberellic acid, thidiazuron.

\section{INTRODUÇÃO}

A videira da cultivar Niagara Rosada tem se apresentado como uma alternativa interessante para a produção de uvas na região Noroeste do Estado de São Paulo. Pouco suscetível às doenças fúngicas, e conseqüentemente com baixo custo de produção, a uva 'Niagara Rosada' atinge preços elevados no período de junho a novembro, entressafra das regiões vitícolas tradicionais do Estado. Entretanto, devido às condições edafoclimáticas, os cachos e bagas são relativamente pequenos, o que é desfavorável à comercialização.

Castro et al. (1974) constataram que aplicações de ácido giberélico $\left(\mathrm{AG}_{3}\right)$ a $100 \mathrm{mg} . \mathrm{L}^{-1}, 11$ dias após florescimento, aumentaram a massa dos cachos, o número e a massa das bagas e a massa da ráquis de uvas da cultivar Niagara Rosada, na região de Jundiaí-SP. Por outro lado, Maraschin et al. (1986) não observaram efeitos de aplicações de $\mathrm{AG}_{3}$ nas doses entre 0 e $120 \mathrm{mg} . \mathrm{L}^{-1}$, nas características físicas dos cachos da cultivar Niagara Branca. Entretanto, doses superiores a $30 \mathrm{mg} . \mathrm{L}^{-1}$ reduziram o teor de sólidos solúveis totais do mosto. Pereira et al. (1979) verificaram para a cultivar Niagara Rosada aumento do comprimento e da largura dos cachos e da massa dos engaços mediante duas aplicações de $\mathrm{AG}_{3}$ a $100 \mathrm{mg} . \mathrm{L}^{-1}$, antes e após o florescimento, porém não houve diferenças significativas para as variáveis massa e número de bagas.

O thidiazuron (N-fenil-N-1,2,3-tidiazol-5-tiuréia) (TDZ) é uma citocinina sintética utilizada na cultura do algodoeiro para provocar desfolhamento, e em cultura de tecidos para induzir brotação in vitro (Petri et al., 1992). Em fruticultura, há trabalhos que comprovaram a eficiência do thidiazuron no aumento do tamanho e pegamento dos frutos de maçãs, kiwis e caquis (Petri et al., 1992; Schuck \& Petri, 1992; Itai et al., 1995).

Nas variedades de videiras sem sementes Sovereign Coronation, Simone, Selection 495 e Selection 535, Reynolds et al. (1992) estudaram os efeitos de aplicações de thidiazuron nas doses de $0 ; 4$ e 8 mg.L. $\mathrm{L}^{-1}$, quando as bagas atingiram $5 \mathrm{~mm}$ de diâmetro. Pelos resultados obtidos, verificaram que o thidiazuron aumentou linearmente a massa dos cachos e das bagas e reduziu o teor de sólidos solúveis totais e o pH do mosto.

Por outro lado, Byun \& Kim (1995) trataram cachos de videiras da cultivar Kyoho, com $\mathrm{AG}_{3}$ a $20 \mathrm{mg} . \mathrm{L}^{-1}$ e thidiazuron a 5 ou $10 \mathrm{mg} . \mathrm{L}^{-1}, 5$ dias após o pleno florescimento, e verificaram que $\mathrm{AG}_{3}$, aumentou o tamanho das bagas, enquanto o thidiazuron aumentou o número de bagas. Entretanto, o thidiazuron reduziu a coloração das bagas e o teor de sólidos solúveis totais.

Tendo em vista as possibilidades de utilização de reguladores vegetais para a melhoria da qualidade de uvas, este trabalho teve como objetivo estudar os efeitos de aplicações de thidiazuron e ácido giberélico nas características dos cachos e bagas de uvas da cultivar Niagara Rosada na região da Nova Alta Paulista.

\section{MATERIALEMÉTODOS}

Este experimento foi conduzido em vinhedo da cultivar Niagara Rosada localizado no município de Junqueirópolis-SP. As videiras, em

\footnotetext{
${ }^{1}$ (Trabalho 166/2003). Recebido: 31/10/2003. Aceito para publicação: 23/03/04 . Parte da Tese de Doutorado do primeiro autor apresentada ao curso de pós-graduação em Agronomia da Faculdade de Ciências Agronômicas - UNESP, Câmpus de Botucatu-SP. (Bolsa da FAPESP)

${ }^{2}$ Eng. Agr. Dr., Prof. Adjunto Departamento de Agronomia, CAA, UNICENTRO. R. Simeão Varela de Sá nº 03, 85035-120 Guarapuava-PR. F: (042) 629-1444. Email: rbotelho@unicentro.br

${ }^{3}$ Eng. Agr. Dr., Pesquisador Científico. Instituto Agronômico de Campinas, Caixa Postal 28, CEP 13001-970 Campinas-SP. E-mail: ejppires@ @ec.iac.br
} 
$3^{\circ}$ ano de produção e enxertadas sobre porta-enxerto IAC-572 'Jales', encontravam-se no espaçamento $3 \times 2 \mathrm{~m}$, e conduzidas no sistema de pérgola.

Para os tratamentos, utilizou-se de thidiazuron (TDZ) a 5 ou $10 \mathrm{mg} . \mathrm{L}^{-1}$ e ácido giberélico a $35 \mathrm{mg} . \mathrm{L}^{-1}$, combinados ou não; aplicados 1 ; 2 ou 3 vezes, em intervalos semanais, a partir dos 14 dias após o pleno florescimento. Desta forma, os tratamentos foram os seguintes:

$$
\begin{aligned}
& \mathrm{T} 1 \text { - Testemunha } \\
& \text { T2 - TDZ a 5mg. } \mathrm{L}^{-1}-1 \text { aplicação } \\
& \text { T3 - TDZ a 5mg.. } \mathrm{L}^{-1}-2 \text { aplicações } \\
& \text { T4 - TDZ a 5mg.L } \mathrm{L}^{-1}-3 \text { aplicações } \\
& \text { T5 - TDZ a 10mg.L } \mathrm{L}^{-1}-1 \text { aplicação } \\
& \text { T6 - TDZ a 10mg. } \mathrm{L}^{-1}-2 \text { aplicações } \\
& \text { T7 - TDZ a 10mg. } \mathrm{L}^{-1}-3 \text { aplicações } \\
& \text { T8 - TDZ a 5mg. } \mathrm{L}^{-1}+\mathrm{AG}_{3} \text { a } 35 \mathrm{mg} . \mathrm{L}^{-1}-1 \text { aplicação } \\
& \text { T9 - TDZ a 5mg. } \mathrm{L}^{-1}+\mathrm{AG}_{3} \text { a } 35 \mathrm{mg} . \mathrm{L}^{-1}-2 \text { aplicações } \\
& \text { T10 - TDZ a 5mg.L } \mathrm{L}^{-1}+\mathrm{AG}_{3} \text { a } 35 \mathrm{mg} . \mathrm{L}^{-1}-3 \text { aplicações } \\
& \text { T11 - TDZ a 10mg. } \mathrm{L}^{-1}+\mathrm{AG}_{3} \text { a } 35 \mathrm{mg} . \mathrm{L}^{-1}-1 \text { aplicação } \\
& \text { T12 - TDZ a 10mg. } \mathrm{L}^{-1}+\mathrm{AG}_{3} \text { a } 35 \mathrm{mg} . \mathrm{L}^{-1}-2 \text { aplicações } \\
& \text { T13 - TDZ a 10mg. } \mathrm{L}^{-1}+\mathrm{AG}_{3} \text { a } 35 \mathrm{mg} . \mathrm{L}^{-1}-3 \text { aplicações } \\
& \text { T14 - } \mathrm{AG}_{3} \text { a } 35 \mathrm{mg} . \mathrm{L}^{-1}-1 \text { aplicação } \\
& \text { T15- } \mathrm{AG}_{3} \text { a } 35 \mathrm{mg} . \mathrm{L}^{-1}-2 \text { aplicações } \\
& \text { T16 }-\mathrm{AG}_{3} \text { a } 35 \mathrm{mg} . \mathrm{L}^{-1}-3 \text { aplicações }
\end{aligned}
$$

Todas as práticas culturais no vinhedo, exceto a utilização de ácido giberélico, foram idênticas ao sistema convencional da propriedade para toda a área experimental.

Os tratamentos com reguladores vegetais foram efetuados através de única imersão dos cachos na solução contida em recipiente plástico, adicionada de espalhante adesivo Iharaguen-S ${ }^{\circledR}$ a $1 \%$, formulação comercial com $20 \%$ de polioxietileno alquilfenol éter. Para o preparo das soluções com reguladores vegetais, foram utilizados os produtos comerciais Dropp ${ }^{\circledR}, \operatorname{com} 50 \%$ de thidiazuron, e Pro-Gibb ${ }^{\circledR}$, com $10 \%$ de ácido giberélico.

O delineamento experimental foi em blocos casualizados, com 7 repetições, e 2 cachos por parcela. Videiras semelhantes em vigor foram selecionadas para cada bloco, numa mesma linha de plantio, e os cachos foram sorteados dentro de cada um dos 7 blocos, para a realização dos tratamentos.

A coleta dos cachos foi realizada aos 118 dias após a poda, em
24 de julho de 2000, quando o tratamento-testemunha atingiu o ponto de colheita comercial, ou seja, com teor de sólidos solúveis totais mínimo de $14^{\circ}$ Brix.

Foram determinadas as seguintes variáveis:

1. Massa dos cachos, bagas e engaços, em balança de precisão. Após a medição da massa dos cachos, as bagas foram separadas dos pedicelos, cortando-os com tesoura, para posterior pesagem das bagas e engaços, separadamente;

2. Comprimento e largura dos cachos e bagas (amostra de 20 bagas), com paquímetro digital;

3. Número de bagas por cacho;

4. Número de sementes por baga (amostra de 20 bagos);

5. Relação comprimento/largura das bagas.

Para cada parcela, foram realizadas as seguintes análises do mosto de 100 bagas:

1. Teor de sólidos solúveis totais: com auxílio de refratômetro de mesa com auto-compensação de temperatura (Carvalho et al., 1990);

2. Acidez total titulável: por titulação em uma alíquota de $5 \mathrm{ml}$ do mosto com $\mathrm{NaOH} 0,1 \mathrm{~N}$, e expressa em g de ácido tartárico por 100ml de mosto (Carvalho et al., 1990);

3. Potencial Hidrogeônico: com auxílio de pHmetro digital (Carvalho et al., 1990);

4. Relação teor de sólidos solúveis totais/acidez total titulável (SST/ATT).

\begin{tabular}{|c|c|c|c|c|}
\hline Tratamentos & Massa Cachos (g) & Comprimento cachos (cm) & Largura cachos (cm) & Massa engaços (g) \\
\hline Testemunha $^{1}$ & $206,6 \quad g^{2}$ & 12,48 & 7,60 & $2,51 \mathrm{c}$ \\
\hline TDZ 5 - 1x & 244,6 bcde & 12,96 & 8,04 & $3,27 \mathrm{ab}$ \\
\hline TDZ $5-2 x$ & $272,2 \mathrm{a}$ & 13,67 & 8,81 & $3,68 \mathrm{a}$ \\
\hline TDZ $5-3 x$ & 239,1 & 13,00 & 8,26 & $3,25 \mathrm{ab}$ \\
\hline TDZ $10-1 \mathrm{x}$ & 244,0 bcdef & 13,74 & 7,94 & $3,62 \mathrm{a}$ \\
\hline TDZ $10-2 x$ & $222,8 \quad$ efg & 13,27 & 8,38 & $2,97 a b c$ \\
\hline TDZ $10-3 x$ & 232,4 def & 13,44 & 8,01 & $3,16 a b c$ \\
\hline $\mathrm{TDZ} 5+\mathrm{AG}_{3}-1 \mathrm{x}$ & $258,1 \mathrm{abc}$ & 14,24 & 8,23 & $3,26 \mathrm{ab}$ \\
\hline $\mathrm{TDZ} 5+\mathrm{AG}_{3}-2 \mathrm{x}$ & 219,3 & 12,73 & 8,04 & $3,10 a b c$ \\
\hline $\mathrm{TDZ} 5+\mathrm{AG}_{3}-3 \mathrm{x}$ & $245,5 \mathrm{abcd}$ & 13,58 & 8,01 & $3,47 \mathrm{a}$ \\
\hline $\mathrm{TDZ} 10+\mathrm{AG}_{3}-1 \mathrm{x}$ & $202,6 \quad \mathrm{~g}$ & 12,10 & 7,47 & $2,70 \mathrm{bc}$ \\
\hline $\mathrm{TDZ} 10+\mathrm{AG}_{3}-2 \mathrm{x}$ & 244,0 bcdef & 12,97 & 8,11 & $3,11 \mathrm{abc}$ \\
\hline $\mathrm{TDZ} 10+\mathrm{AG}_{3}-3 \mathrm{x}$ & $266,2 \mathrm{ab}$ & 13,77 & 8,63 & $3,57 \mathrm{a}$ \\
\hline $\mathrm{AG}_{3}-1 \mathrm{x}$ & 230,5 def & 12,97 & 7,70 & $2,54 \mathrm{c}$ \\
\hline $\mathrm{AG}_{3}-2 \mathrm{x}$ & 224,9 cdefg & 13,47 & 7,66 & $3,01 \mathrm{abc}$ \\
\hline $\mathrm{AG}_{3}-3 \mathrm{x}$ & 241,5 cdef & 13,07 & 8,67 & $3,26 \mathrm{ab}$ \\
\hline C.V. $(\%)$ & 8,45 & 9,46 & 12,44 & 18,01 \\
\hline
\end{tabular}

Os resultados foram submetidos à análise de variância e comparação de médias, pelo teste de Duncan, ao nível de 5\% de probabilidade.

\section{RESULTADOSE DISCUSSÃO}

A maioria dos tratamentos com reguladores vegetais aumentou significativamente a massa dos cachos (Tabela 1). O maior aumento da massa dos cachos foi verificado para o tratamento com TDZ a $5 \mathrm{mg} . \mathrm{L}^{-1}$, em 2 aplicações, o qual não diferiu significativamente dos seguintes tratamentos: TDZ 5 mg.L $\mathrm{L}^{-1}+\mathrm{AG}_{3}$, em 1 aplicação; TDZ 5 mg.L $\mathrm{L}^{-1}+\mathrm{AG}_{3}$, em 3 aplicações, e TDZ $10 \mathrm{mg} . \mathrm{L}^{-1}+\mathrm{AG}_{3}$, em 3 aplicações. Aumento da massa dos cachos de uvas tratadas com ácido giberélico ou thidiazuron foi também verificado por outros autores (Castro et al., 1974; Pereira et al., 1979; Reynolds et al., 1992 e Byun \& Kim, 1995).

TABELA 1 - Massa, comprimento e largura dos cachos, e massa dos engaços de uva 'Niagara Rosada' tratada com thidiazuron e ácido giberélico (Junqueirópolis-SP, 2000).

${ }^{1} \mathrm{TDZ} 5=$ thidiazuron $5 \mathrm{mg} \cdot \mathrm{L}^{-1} ; \mathrm{TDZ} 10=$ thidiazuron $10 \mathrm{mg} \cdot \mathrm{L}^{-1} ; \mathrm{AG}_{3}=$ ácido giberélico $35 \mathrm{mg} \cdot \mathrm{L}^{-1}$

${ }^{2}$ Médias seguidas pela mesma letra, na mesma coluna, não diferem entre si, pelo teste de Duncan, ao nível de $5 \%$ de probabilidade. 
TABELA 2 - Número, massa, comprimento, largura e relação comprimento/largura das bagas de uva 'Niagara Rosada' tratada com thidiazuron e ácido giberélico (Junqueirópolis-SP, 2000).

\begin{tabular}{|c|c|c|c|c|c|}
\hline Tratamentos & Número bagas & Massa bagas (g) & Comprimento bagas (mm) & Largura bagas (mm) & Relação C/L \\
\hline Testemunha $^{1}$ & 58,4 & $3,57 \quad \mathrm{c}$ & 19,15 & $17,13 \quad \mathrm{~d}$ & 1,12 \\
\hline TDZ $5-1 \mathrm{x}$ & 65,3 & $3,68 \mathrm{bc}$ & 19,60 & $17,28 \mathrm{bcd}$ & 1,13 \\
\hline TDZ $5-2 \mathrm{x}$ & 63,3 & $4,37 \mathrm{a}$ & 20,26 & $18,14 \mathrm{abc}$ & 1,11 \\
\hline TDZ $5-3 x$ & 61,6 & $3,86 \mathrm{abc}$ & 19,70 & $17,42 \mathrm{bcd}$ & 1,13 \\
\hline TDZ $10-1 \mathrm{x}$ & 61,6 & $3,93 \mathrm{abc}$ & 20,04 & $17,78 \mathrm{abcd}$ & 1,12 \\
\hline TDZ $10-2 x$ & 54,3 & $4,14 \mathrm{abc}$ & 19,97 & $18,28 \mathrm{a}$ & 1,09 \\
\hline TDZ $10-3 x$ & 61,0 & $3,81 \mathrm{abc}$ & 19,57 & $17,25 \mathrm{~cd}$ & 1,13 \\
\hline $\mathrm{TDZ} 5+\mathrm{AG}_{3}-1 \mathrm{x}$ & 66,6 & $3,82 \mathrm{abc}$ & 19,61 & $17,44 \mathrm{abcd}$ & 1,12 \\
\hline $\mathrm{TDZ} 5+\mathrm{AG}_{3}-2 \mathrm{x}$ & 55,3 & $3,94 a b c$ & 20,05 & $17,68 \mathrm{abcd}$ & 1,14 \\
\hline $\mathrm{TDZ} 5+\mathrm{AG}_{3}-3 \mathrm{x}$ & 58,3 & $4,26 \mathrm{ab}$ & 20,14 & $18,18 \mathrm{ab}$ & 1,10 \\
\hline $\mathrm{TDZ} 10+\mathrm{AG}_{3}-1 \mathrm{x}$ & 54,3 & $4,09 \mathrm{abc}$ & 20,24 & $17,69 \mathrm{abcd}$ & 1,12 \\
\hline $\mathrm{TDZ} 10+\mathrm{AG}_{3}-2 \mathrm{x}$ & 61,0 & $3,95 \mathrm{abc}$ & 19,86 & $17,68 \mathrm{abcd}$ & 1,12 \\
\hline $\mathrm{TDZ} 10+\mathrm{AG}_{3}-3 \mathrm{x}$ & 60,7 & $4,38 \mathrm{a}$ & 20,51 & $18,28 \mathrm{a}$ & 1,12 \\
\hline $\mathrm{AG}_{3}-1 \mathrm{x}$ & 58,0 & $3,89 \mathrm{abc}$ & 20,04 & $17,82 \mathrm{abcd}$ & 1,12 \\
\hline $\mathrm{AG}_{3}-2 \mathrm{x}$ & 58,6 & $3,82 \mathrm{abc}$ & 19,63 & $17,44 \mathrm{abcd}$ & 1,12 \\
\hline $\mathrm{AG}_{3}-3 \mathrm{x}$ & 62,4 & $3,86 a b c$ & 19,64 & $17,26 \mathrm{~cd}$ & 1,12 \\
\hline C.V. $(\%)$ & 14,0 & 12,35 & 4,04 & 4,09 & 1,96 \\
\hline
\end{tabular}

${ }^{1}$ TDZ 5 = thidiazuron $5 \mathrm{mg} . \mathrm{L}^{-1}$; TDZ 10 = thidiazuron $10 \mathrm{mg} . \mathrm{L}^{-1} ; \mathrm{AG}_{3}=$ ácido giberélico $35 \mathrm{mg} . \mathrm{L}^{-1}$

${ }^{2}$ Médias seguidas pela mesma letra, na mesma coluna, não diferem entre si, pelo teste de Duncan, ao nível de 5\% de probabilidade

Não houve diferenças significativas para as variáveis comprimento e largura dos cachos, embora a maioria dos tratamentos com reguladores vegetais tenha apresentado valores superiores ao da testemunha (Tabela 1). Medições de volume ou circunferência dos cachos talvez poderiam demonstrar de forma mais evidente o aumento do tamanho dos cachos.

A massa dos engaços foi aumentada para a maioria dos tratamentos com reguladores vegetais (Tabela 1). O maior aumento para esta variável foi para o tratamento com TDZ a 5mg.L $\mathrm{L}^{-1}$, em 2 aplicações, que incrementou a massa dos engaços em 46,6\% em relação à testemunha, sem, contudo, prejudicar a aparência dos cachos. Estes resultados são condizentes com aqueles apresentados por Castro et al. (1974), Pereira et al. (1979) e Byun \& Kim (1995).

Poucos tratamentos aumentaram significativamente a massa e a largura das bagas, e não houve diferenças significativas para a variável comprimento das bagas (Tabela 2). No entanto, todos os tratamentos com reguladores vegetais apresentaram valores absolutos superiores ao da testemunha para estas variáveis. Os tratamentos com reguladores vegetais também não influenciaram significativamente no número de bagas por cacho (Tabela 2 ).
Para o aumento da massa e das dimensões das bagas, destacaram-se os tratamentos com TDZ a $5 \mathrm{mg} . \mathrm{L}^{-1}$, em 2 aplicações, e com TDZ a 10mg. $\mathrm{L}^{-1}+\mathrm{AG}_{3}$, em 3 aplicações. Os tratamentos com thidiazuron a 5mg. $\mathrm{L}^{-1}$, em duas aplicações, e o tratamento com TDZ a 10 $\mathrm{mg} \cdot \mathrm{L}^{-1}+\mathrm{AG}_{3}$, em três aplicações, apresentaram massa média das bagas de 4,37 e 4,38g, respectivamente, representando um aumento de cerca de $22,4 \%$ em relação à testemunha. Não se constatou influência dos reguladores vegetais na relação comprimento/largura das bagas (Tabela 2).

Aumento do tamanho das bagas de uvas tratadas com thidiazuron foi também constatado por Reynolds et al. (1992), em 4 cultivares de uvas sem sementes. O maior desenvolvimento das bagas e engaços promovido por aplicações de thidiazuron pode ser explicado pela sua ação de citocinina em tecidos vegetais, induzindo a divisão celular, em geral, por uma interação com auxinas (Mcgraw, 1988). Além disso, segundo Davies (1988), citocininas podem também estimular o crescimento celular e retardar a senescência de órgãos vegetais.

Aplicações isoladas de ácido giberélico foram pouco efetivas no aumento do tamanho das bagas de uvas da cultivar Niagara Rosada, estando de acordo com os relatos de Pereira et al. (1979) e Maraschin et

TABELA 3 - Teor de sólidos solúveis totais, acidez total titulável, relação sólidos solúveis totais/acidez total titulável e pH do mosto, e número de sementes de uva 'Niagara Rosada' tratada com thidiazuron e ácido giberélico (Junqueirópolis-SP, 2000).

\begin{tabular}{|c|c|c|c|c|c|}
\hline Tratamentos & SST (\%) & ATT $(\mathrm{g} / 100 \mathrm{ml})$ & Relação SST/ATT & pH & Número sementes \\
\hline Testemunha $^{1}$ & $16,56 \mathrm{abcd}^{2}$ & 1,059 & $15,67 a b c$ & 3,229 & 3,65 \\
\hline TDZ $5-1 \mathrm{x}$ & $17,14 \mathrm{ab}$ & 1,002 & $17,27 \mathrm{a}$ & 3,201 & 3,58 \\
\hline TDZ $5-2 x$ & $16,26 \mathrm{bcd}$ & 1,122 & $14,58 \mathrm{c}$ & 3,140 & 3,63 \\
\hline TDZ $5-3 x$ & $15,67 \mathrm{~cd}$ & 1,116 & $14,54 \mathrm{c}$ & 3,257 & 3,63 \\
\hline TDZ $10-1 x$ & $17,23 \mathrm{ab}$ & 1,066 & $16,21 \mathrm{abc}$ & 3,210 & 3,54 \\
\hline TDZ $10-2 x$ & $17,04 \mathrm{ab}$ & 1,127 & $15,25 \mathrm{abc}$ & 3,188 & 3,34 \\
\hline TDZ $10-3 x$ & $16,61 \mathrm{abcd}$ & 1,082 & $15,48 \mathrm{abc}$ & 3,174 & 3,40 \\
\hline $\mathrm{TDZ} 5+\mathrm{AG}_{3}-1 \mathrm{x}$ & $16,56 \mathrm{abcd}$ & 1,050 & $15,81 \mathrm{abc}$ & 3,194 & 3,51 \\
\hline $\mathrm{TDZ} 5+\mathrm{AG}_{3}-2 \mathrm{x}$ & $17,53 \mathrm{a}$ & 1,088 & $16,11 \mathrm{abc}$ & 3,179 & 3,64 \\
\hline $\mathrm{TDZ} 5+\mathrm{AG}_{3}-3 \mathrm{x}$ & $16,36 \mathrm{abcd}$ & 1,124 & $14,58 \mathrm{c}$ & 3,216 & 3,64 \\
\hline $\mathrm{TDZ} 10+\mathrm{AG}_{3}-1 \mathrm{x}$ & $17,16 \mathrm{ab}$ & 1,082 & $16,00 \mathrm{abc}$ & 3,203 & 3,40 \\
\hline $\mathrm{TDZ} 10+\mathrm{AG}_{3}-2 \mathrm{x}$ & $16,50 \mathrm{abcd}$ & 1,092 & $15,24 a b c$ & 3,231 & 3,47 \\
\hline $\mathrm{TDZ} 10+\mathrm{AG}_{3}-3 \mathrm{x}$ & $15,54 \quad d$ & 1,124 & $13,95 \mathrm{c}$ & 3,148 & 3,51 \\
\hline $\mathrm{AG}_{3}-1 \mathrm{x}$ & $16,86 \mathrm{abcd}$ & 1,102 & $15,28 \mathrm{abc}$ & 3,193 & 3,41 \\
\hline $\mathrm{AG}_{3}-2 \mathrm{x}$ & $16,83 \mathrm{abc}$ & 1,000 & $17,16 \mathrm{ab}$ & 3,208 & 3,37 \\
\hline $\mathrm{AG}_{3}-3 \mathrm{x}$ & $16,18 \mathrm{bcd}$ & 1,108 & $14,87 \mathrm{bc}$ & 3,121 & 3,53 \\
\hline C.V. $(\%)$ & 5,92 & 8,35 & 12,03 & 3,41 & 7,69 \\
\hline
\end{tabular}

${ }^{1} \mathrm{TDZ} 5=$ thidiazuron $5 \mathrm{mg} \cdot \mathrm{L}^{-1} ; \mathrm{TDZ} 10=$ thidiazuron $10 \mathrm{mg} \cdot \mathrm{L}^{-1} ; \mathrm{AG}_{3}=$ ácido giberélico $35 \mathrm{mg} \cdot \mathrm{L}^{-1}$

${ }^{2}$ Médias seguidas pela mesma letra, na mesma coluna, não diferem entre si, pelo teste de Duncan, ao nível de $5 \%$ de probabilidade. 
al. (1986).

O teor de sólidos solúveis totais apresentou diferenças significativas entre 1 e 3 aplicações, para os tratamentos com TDZ a 5mg.L-1 ${ }^{-1}$ e com TDZ a 10mg.L $\mathrm{L}^{-1}+\mathrm{AG}_{3}$. Redução do teor de sólidos solúveis totais do mosto de uvas tratadas com thidiazuron ou ácido giberélico foi também constatada por Maraschin et al. (1986), Reynolds et al. (1992) e por Byun e Kim (1995).

Pelos resultados aqui apresentados, ficou evidente a possibilidade de utilização de reguladores vegetais para incrementar o tamanho e massa dos cachos e bagas de uvas 'Niagara Rosada' na região da Nova Alta Paulista. O tratamento com thidiazuron a $5 \mathrm{mg} . \mathrm{L}^{-1}$, em 2 aplicações, foi suficiente para a obtenção de cachos e bagas com maior desenvolvimento e, portanto, com qualidade superior para a comercialização (Figura 1). Para as condições deste experimento, este tratamento possibilitou aumento de $31,7 \%$ da massa dos cachos e de $22,4 \%$ da massa das bagas, sem alterar o teor de sólidos solúveis totais, a acidez total titulável, o pH e a relação SST/ATT do mosto.

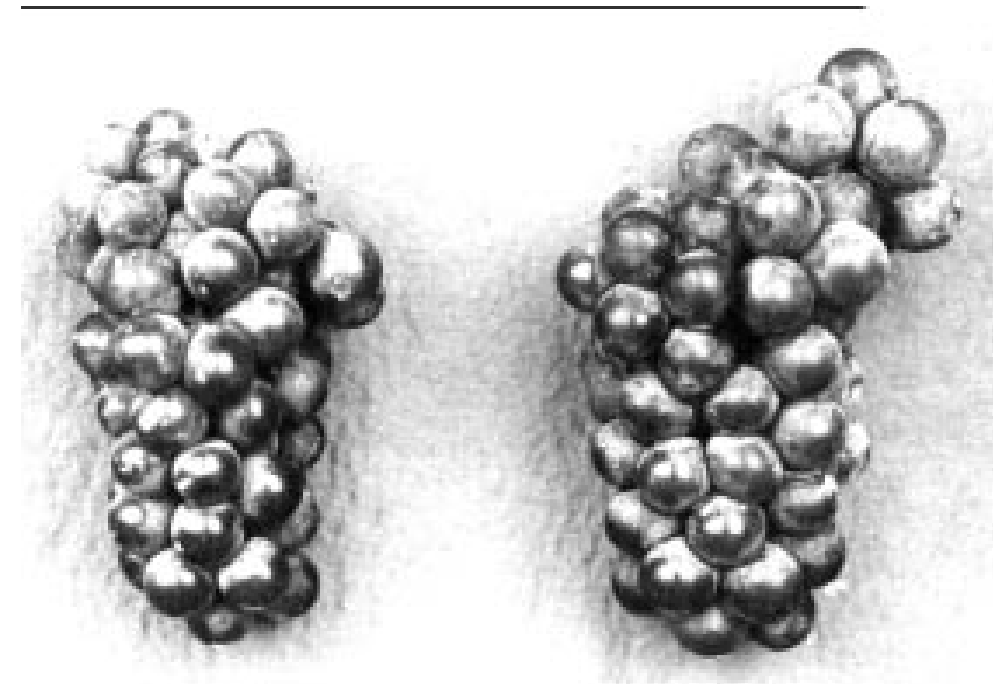

FIGURA 1 - Cachos de uva 'Niagara Rosada' do tratamento-testemunha (esquerda) e tratados com thidiazuron a $5 \mathrm{mg} . \mathrm{L}^{-1}$ (direita), aos 14 e 21 dias após o pleno florescimento.

O thidiazuron ainda não está registrado para a cultura da videira no Brasil, não podendo ser recomendado para o uso comercial. Novas pesquisas deverão ser conduzidas, incluíndo estudos toxicológicos, objetivando a futura utilização desta substância para a melhoria da qualidade de uvas 'Niagara Rosada', uma vez que o ácido giberélico usado isoladamente não apresentou resultados satisfatórios.

\section{CONCLUSÕES}

1) Aplicações de thidiazuron associado ou não ao ácido giberélico após florescimento foram efetivas no aumento da massa e dimensões das bagas de uvas 'Niagara Rosada', sem alterar o teor de sólidos solúveis totais, a acidez total titulável e o pH do mosto.
2) Duas aplicações de thidiazuron a $5 \mathrm{mg} . \mathrm{L}^{-1}$ foram efetivas no aumento do tamanho das bagas de uvas 'Niagara Rosada', embora não tenham apresentado aumentos significativos nas dimensões dos cachos.

\section{AGRADECIMENTOS}

Agradecimentos aos técnicos do Instituto Agronômico de Campinas, o Sr. Valdeir Biundes Hermoso e a Sra. Maria das Graças dos Santos Lima.

\section{REFERÊNCIASBIBLIOGRÁFICAS}

BYUN, J.K.; KIM, J.S. Effects of GA 3 , thidiazuron and ABA on fruit set and quality of 'Kyoho' grapes. Journal of the Korean Society for Horticultural Science, Kyongsan, v.36, n.2, p.231-239, 1995.

CARVALHO, C.R.L.; CARVALHO, P.R.N.; MANTOVANI, D.M.B.; MORAES, R.M. Análise química de alimentos. Campinas: ITAL, 1990. 121p.

CASTRO, P.R.C.; FERRAZ, E.C.; SCARANARI, H.J. Efeitos de giberelinas e auxina na frutificação da videira 'Niagara Rosada'. Anais da E.S.A. “Luiz de Queiroz”, v.31, p.367-383, 1974.

DAVIES, P.J. The plant hormones: their nature, occurrence, and functions. In: _. Plant hormones and their role in plant growth and development. 2. ed. Dordrecht: Kluwer Academic Publishers, 1988. p.1-11.

ITAI, A.; TANABE, K.; TAMURA, F.; SUSAKI, S.; YONEMORI, K.; SUGIURA, A. Synthetic cytokinins control persimmon fruit shape, size and quality. Journal of Horticultural Science, Ashford, v.70, n.6, p.867-873, 1995.

MACGRAW, B.A. Cytokinin biosynthesis and metabolism. In: DAVIES, P.J. Plant hormones and their role in plant growth and development. 2. ed. Dordrecht: Kluwer Academic Publishers, 1988. p.76-93.

MARASCHIN, M.; GUERRA, M.P.; SILVA, A.L. Efeitos do ácido giberélico e ethephon sobre as características dos cachos e frutos da cv. Niagara Branca (Vitis labrusca L.) Revista Brasileira de Fruticultura, Cruz das Almas, v.8, n.2, p.51-57, 1986.

PEREIRA, F.M.; SIMÃO, S.; MARTINS, F.P.; IGUE, T. Efeitos da giberelina sobre cachos da cultivar de videira Niagara Rosada. Científica, Jaboticabal, v.7, n.1, p.53-58, 1979.

PETRI, J.L.; ARGENTA, L.C.; SUZUKI, A. Efeitos do thidiazuron no tamanho e desenvolvimento dos frutos da macieira. Revista Brasileira de Fruticultura, Cruz das Almas, v.14, n.2, p.127-134, 1992.

REYNOLDS, A.G.; WARDLE, D.A.; ZUROWSKI, C.; LOONEY, N.E. Phenylureas CPPU and thidiazuron affect yield components, fruit composition, and storage potential of four seedless grape selections. Journal of the American Society for Horticultural Science, Alexandria, v.117, n.1, p.85-89, 1992.

SCHUCK, E.; PETRI, J.L. Efeitos do thidiazuron no peso médio dos frutos de quivi. Revista Brasileira de Fruticultura, Cruz das Almas, v.14, n.2, p.185-188, 1992. 